\title{
THE PROBABILITY AND SEVERITY OF RUIN \\ IN FINITE AND INFINITE TIME
}

\author{
By David C. M. Dickson and Howard R. Waters \\ Heriot-Watt University, Edinburgh
}

\begin{abstract}
In this paper we present algorithms to calculate the probability and severity of ruin in both finite and infinite time for a discrete time risk model. We show how the algorithms can be applied to give approximate values for the same quantities in the classical continuous time risk model.
\end{abstract}

\section{KEYWORDS}

Probability of ruin; severity of ruin; recursive calculation; finite time; infinite time.

\section{INTRODUCTION}

The probability and severity of ruin for the classical continuous time risk model was first discussed in a paper by GERBER et al. (1987). Using their notation, $G(u, y)$ represents the probability that ruin occurs given initial surplus $u$ and that the deficit at the time of ruin is less than $y$. In that paper, general equations for $G(u, y)$ are derived and explicit solutions are found for certain individual claim amount distributions. This work was extended by Gerber and DUfresne (1988) who found explicit solutions for $G(u, y)$ when individual claim amounts were distributed as a combination of exponential distributions. DiCKSON (1989) showed that approximate values of $G(u, y)$ could be calculated by a recursive method by defining a relationship between survival probabilities and the density of $G(u, y)$.

DICKSON and WATERS (1991) present a recursive algorithm to calculate survival probabilities for a discrete time risk model which can be used to approximate to survival probabilities in the classical continuous time risk model. In the present paper, we show how these methods can be adapted to calculate approximate values of $G(u, y)$.

We also present a recursive algorithm for the approximate calculation of the probability and severity of ruin in finite time. Our algorithm is derived from an algorithm presented by DE VYLDER and GoovaERTS (1988) for the approximate calculation of finite time ruin probabilities. 


\section{PRELIMINARIES}

In this paper we are interested in the classical continuous time risk model defined as follows. The claim number process is a Poisson process and individual claim amounts are identically distributed and are independent both of each other and of the number of claims. It is assumed that individual claim amounts are non-negative and have finite mean. Without loss of generality we assume that the Poisson parameter is 1 and that the mean individual claim amount is also 1. Premiums are assumed to be received continuously at a constant rate. Premiums are calculated using a loading factor $\theta(>0)$ so that the premium income per unit time is $(1+\theta)$. The stochastic process $\left\{_{0} Z(t)\right\}_{t \geq 0}$ denotes the surplus at time $t$, given an initial surplus ${ }_{0} Z(0)$, which we denote $u$. (We use the subscript " 0 " to indicate that we are dealing with our initial model.) The time until ruin for this process, denoted ${ }_{0} T$, is defined as follows:

$$
\begin{aligned}
{ }_{0} T & =\inf \left\{t:{ }_{0} Z(t)<0, t>0\right\} \\
& =\infty \text { if }{ }_{0} Z(t) \geqslant 0 \text { for all } t>0
\end{aligned}
$$

We are particularly interested in the probability and severity of ruin for this process, i.e. the probability that ruin occurs and that the surplus at the time of ruin does not go below a given amount, say $-y$, where $y>0$. We denote this probability ${ }_{0} G(u, y)$ and define it as follows:

$$
{ }_{0} G(u, y)=P\left[{ }_{0} T<\infty \text { and }-{ }_{0} Z\left({ }_{0} T\right)<y\right]
$$

We define the probability and severity of ruin in finite time, denoted ${ }_{0} G(u, y, t)$, as follows:

$$
{ }_{0} G(u, y, t)=P\left[{ }_{0} T \leq t \text { and }-{ }_{0} Z\left({ }_{0} T\right)<y\right]
$$

The probability of ruin in finite time for this process is denoted ${ }_{0} \psi(u, t)$ and is defined as follows:

$$
{ }_{0} \psi(u, t)=P\left[{ }_{0} T \leq t\right]
$$

Our objectives in this paper are to obtain numerical values for, or at least approximations to, ${ }_{0} G(u, y)$ and ${ }_{0} G(u, y, t)$. To do this we consider a second risk model which is still a compound Poisson risk model and can be regarded as a rescaled and discretized approximation to the initial risk model. The characteristics of this second model are:

(2.1) - individual claim amounts are distributed on the non-negative integers and have mean $\beta$, where $\beta(>1)$ is an integer,

- the Poisson parameter for the expected number of claims per unit time is $1 /[(1+\theta) \beta]$,

- the premium income per unit time is 1 .

The method for constructing this second risk model and the reasons why it can be regarded as an approximation to the initial model are discussed fully by 
DiCKSON and WATERS (1991, Section 1). The discrete time stochastic process $\{Z(n)\}_{n=0}^{\infty}$ denotes the surplus at time $n$ for the model specified by $(2.1)$ given an initial surplus of $Z(0)=u$, which we shall always assume to be a non-negative integer. An important feature of this model is that the aggregate claims in each unit of time are distributed on the non-negative integers so that in one unit of time the surplus can increase by 1 (if the aggregate claims are 0 ), stay at the same level (if the aggregate claims are 1) or decrease by an integer amount. The (discrete) time until ruin for this model is denoted $T$ and defined as follows:

$$
\begin{aligned}
T & =\min \{n: Z(n) \leqslant 0, n=1,2, \ldots\} \\
& =\infty \text { if } Z(n)>0 \text { for } n=1,2, \ldots
\end{aligned}
$$

Note that we regard hitting zero as being ruined. This is because we are approximating continuous time probabilities by discrete time probabilities. If the surplus in discrete time hits zero then, with probability one, it was below zero in continuous time immediately prior to passing through zero. DICKSON and WATERs (1991, Section 8) discuss this point at greater length. Note also that if the initial surplus is 0 then ruin does not occur at time 0 but will occur at time 1 unless the aggregate claims in the first time period are 0 . The probabilities of ruin in finite time and in infinite time for this process are denoted $\psi(u, t)$ and $\psi(u)$ respectively and are defined as follows:

$$
\begin{aligned}
& \psi(u, t)=P[T \leq t] \\
& \psi(u)=P[T<\infty]
\end{aligned}
$$

The probability and severity of ruin in infinite and finite time for the model specified by $(2.1)$ are denoted $G(u, y)$ and $G(u, y, t)$, respectively, where $y$ is a positive integer, and are defined as follows:

$$
\begin{aligned}
& G(u, y)=P[T<\infty \text { and }-Z(T)<y] \\
& G(u, y, t)=P[T \leq t \quad \text { and } \quad-Z(T)<y]
\end{aligned}
$$

For any choice of values of $u, y, t$ and $\beta$ such that $\beta, y \beta$ and $t \beta(1+\theta)$ are positive integers and $u \beta$ is a non-negative integer, we regard $\psi(u \beta, t \beta(1+\theta))$, $G(u \beta, y \beta)$ and $G(u \beta, y \beta, t \beta(1+\theta))$ as approximations to ${ }_{0} \psi(u, t),{ }_{0} G(u, y)$ and ${ }_{0} G(u, y, t)$, respectively, for the reasons given by DICKSON and WATERS (1991, Section 1). In general, the larger the value of $\beta$, the better we would expect this approximation to be.

In the following section we present an algorithm which can be used to calculate $G(u, y)$ and in Section 4 we present some numerical results illustrating the use of this algorithm. In Section 5 we present an algorithm for the calculation of $G(u, y, t)$ and finally in Section 6 we present some numerical results illustrating the use of this second algorithm.

Before doing this we need some more notation. For $k=1,2, \ldots$ we denote by $f_{k}$ and $F(k)$ the probabilities that the aggregate claims in a single time period are equal to $k$ and are less than or equal to $k$, respectively. Note that $f_{k}$ can be calculated using PANJER's (1981) recursion formula since the aggregate 
claims have a compound Poisson distribution with individual claim amounts distributed on the non-negative integers.

\section{THE PROBABILITY AND SEVERITY OF RUIN IN INFINITE TIME}

By considering aggregate claims in the first time unit we can write

$$
\begin{aligned}
G(u, y) & =\sum_{j=0}^{u} f_{j} G(u+1-j, y)+\sum_{j=u+1}^{u+y} f_{j} \text { for } u=1,2,3, \ldots \\
& =\sum_{j=0}^{u} f_{j} G(u+1-j, y)+F(u+y)-F(u)
\end{aligned}
$$

Hence

$$
G(u+1, y)=f_{0}^{-1}\left(G(u, y)-\sum_{j=1}^{u} f_{j} G(u+1-\mathrm{j}, y)+F(u)-F(u+y)\right)
$$

for $u=1,2,3, \ldots$.

Thus we can calculate values of $G(u, y)$ recursively from (3.1) provided that we can calculate $G(0, y)$, since

$$
G(0, y)=f_{0} G(1, y)+F(y)-F(0)
$$

and hence

$$
G(1, y)=f_{0}^{-1}(G(0, y)+F(0)-F(y))
$$

We can find $G(0, y)$ by using a method similar to that of DUFRESNE (1988, Section 3). For a fixed value of $y$, define

$$
\begin{aligned}
& d(0)=G(0, y) \text { and } \\
& d(u)=G(u, y)-G(u-1, y) \text { for } u=1,2,3, \ldots
\end{aligned}
$$

Then

$$
\begin{aligned}
d(u) & =\sum_{j=0}^{u-1} f_{j} d(u+1-j)+f_{u} G(1, y)+f_{u+y}-f_{u} \text { for } u=1,2,3, \ldots \\
& =\sum_{j=0}^{u} f_{j} d(u+1-j)+f_{u+y}-f_{u}+f_{u} d(0)
\end{aligned}
$$

Also, note that

$$
\begin{aligned}
& G(0, y)=d(0)=f_{0} G(1, y)+F(y)-F(0) \\
& =f_{0} d(1)+f_{0} d(0)+F(y)-F(0)
\end{aligned}
$$

Now define $J(s)=\sum_{x=0}^{\infty} s^{x} d(x)$ and $H(s)=\sum_{x=0}^{\infty} s^{x} f_{x}$. 
THE PROBABILITY AND SEVERITY OF RUIN IN FINITE AND INFINITE TIME 181

Note that both these generating functions exist for $|s| \leq 1$.

$$
\begin{aligned}
& \text { Then } J(s)=d(0)+\sum_{x=1}^{\infty} s^{x} d(x) \\
& =\sum_{x=1}^{\infty} s^{x}\left(f_{x} d(0)+\sum_{j=0}^{x} f_{j} d(x+1-j)\right)+\sum_{x=1}^{\infty} s^{x}\left(f_{x+y}-f_{x}\right) \\
& \quad+f_{0} d(1)+f_{0} d(0)+F(y)-f_{0}
\end{aligned}
$$

(using (3.2) and (3.3))

$$
\begin{aligned}
& =\sum_{x=0}^{\infty} s^{x} \sum_{j=0}^{x} f_{j} d(x+1-j)+\sum_{x=0}^{\infty} s^{x} f_{x} d(0)+\sum_{x=1}^{\infty} s^{x} f_{x+y}-\sum_{x=0}^{\infty} s^{x} f_{x}+F(y) \\
& =\sum_{x=0}^{\infty} s^{x} \sum_{j=0}^{x} f_{j} d(x+1-j)+(d(0)-1) H(s)+\sum_{x=0}^{\infty} s^{x} f_{x+y}+F(y-1) \\
& =s^{-1} H(s)(J(s)-d(0))+(d(0)-1) H(s)+\sum_{x=0}^{\infty} s^{x} f_{x+y}+F(y-1)
\end{aligned}
$$

and hence

$$
J(s)=\frac{\sum_{x=0}^{\infty} s^{x} f_{x+y}+F(y-1)-s^{-1} H(s) d(0)+(d(0)-1) H(s)}{1-s^{-1} H(s)}
$$

Now note that $J(1)=\lim _{x \rightarrow \infty} G(x, y)=0$, and that $H(1)=1$. Hence we can find $d(0)$ from (3.4) by setting $s=1$. With $s=1$, both the numerator and denominator of (3.4) are zero, so by applying L'Hôspital's rule we find that

$$
\begin{aligned}
& \begin{aligned}
d(0) & =\sum_{x=0}^{\infty} x f_{x}-\sum_{x=0}^{\infty} x f_{x+y} \\
& =\sum_{j=1}^{y} j f_{j}+y \sum_{j=y+1}^{\infty} f_{j}=\sum_{j=0}^{y-1}(1-F(j))
\end{aligned} \\
& \text { i.e. } \quad G(0, y)=\sum_{j=0}^{y-1}(1-F(j))
\end{aligned}
$$

Hence we can calculate $G(0, y)$ as it is expressed in terms of the distribution function of aggregate claims per unit time. Note that by letting $y \rightarrow \infty$ on the 
right hand side of (3.5) we find the mean of the distribution, i.e. $1 /(1+\theta)$. As $\lim _{y \rightarrow \infty} G(0, y)=\psi(0)$, we see that the limiting form of (3.5) is consistent with the expression for the survival probability from initial surplus zero given by DiCKSON and WATERS (1991, Section 7). It is interesting to compare our expression for $G(0, y)$ with that for ${ }_{0} G(0, y)$. Integrating equation (4) of GERBER et al. (1987) we find that, using their notation

$$
{ }_{0} G(0, y)=\frac{\lambda}{c} \int_{0}^{y}(1-P(\mathrm{x})) d x
$$

where $\lambda$ is the Poisson parameter, $c$ is the premium income per unit time and $P(x)$ is the distribution function of individual claim amounts. The right hand side of (3.6) equals the expected retained aggregate claim amount per unit time under excess of loss reinsurance with retention level $y$, divided by the premium income per unit time before reinsurance. The right hand side of (3.5) gives the expected retained aggregate claim amount per unit time under stop loss reinsurance with retention level $y$, divided by premium income per unit time (i.e. 1) before reinsurance. By making this comparison, it is reasonable to expect that, for a large value of $\beta, G(0, y \beta)$ will give a good approximation to ${ }_{0} G(0, y)$. The reason for this is that for a large value of $\beta$, the probability of more than one claim per unit time will be very small and hence stop loss reinsurance will be virtually identical to excess of loss reinsurance. For example, if $\beta=100$ and $\theta=0.1$, then the probability of more than one claim per unit time is 0.00004 .

\section{NUMERICAL EXAMPLES}

Tables 1 and 2 show exact and approximate values for ${ }_{0} G(u, y)$ when the individual claim amount distribution is exponential. For this distribution, we can apply the methods of GERBER et al. (1987) to show that

$$
{ }_{0} G(u, y)=\frac{1}{1+\theta} \exp \{-\theta u /(1+\theta)\}(1-\exp \{-y\})
$$

In Table 1 , the premium loading factor, $\theta$, is $10 \%$ and in Table 2 it is $20 \%$. The key for these tables is as follows:

(1) denotes the exact value of ${ }_{0} G(u, y)$,

(2) denotes the approximation to ${ }_{0} G(u, y)$, calculated using (3.5) and (3.1), with the parameter $\beta=50$,

(3) denotes the ratio of the value in (2) to that in (1),

(4) as (2), but with $\beta=100$,

(5) denotes the ratio of the value in (4) to that in (1). 
THE PROBABILITY AND SEVERITY OF RUIN IN FINITE AND INFINITE TIME 183

TABLE 1

INDIVIDUAL CLAIM AMOUNTS ARE EXPONENTIALLY DISTRIBUTED AND THE PREMIUM LOADING FACTOR IS 0.1

\begin{tabular}{|c|c|c|c|c|c|}
\hline & & $\iota=0$ & $u=20$ & $u=60$ & $u=100$ \\
\hline$y=1$ & $\begin{array}{l}(1) \\
(2) \\
(3) \\
(4) \\
(5)\end{array}$ & $\begin{array}{l}0.57466 \\
0.57162 \\
0.99473 \\
0.57314 \\
0.99736\end{array}$ & $\begin{array}{l}0.09328 \\
0.09279 \\
0.99480 \\
0.09303 \\
0.99738\end{array}$ & $\begin{array}{l}0.00246 \\
0.00245 \\
0.99491 \\
0.00245 \\
0.99741\end{array}$ & $\begin{array}{l}0.00006 \\
0.00006 \\
0.99498 \\
0.00006 \\
0.99743\end{array}$ \\
\hline$y=3$ & $\begin{array}{l}(1) \\
(2) \\
(3) \\
(4) \\
(5)\end{array}$ & $\begin{array}{l}0.86383 \\
0.86259 \\
0.99857 \\
0.86321 \\
0.99928\end{array}$ & $\begin{array}{l}0.14022 \\
0.14003 \\
0.99863 \\
0.14012 \\
0.99930\end{array}$ & $\begin{array}{l}0.00369 \\
0.00369 \\
0.99874 \\
0.00369 \\
0.99933\end{array}$ & $\begin{array}{l}0.00010 \\
0.00010 \\
0.99883 \\
0.00010 \\
0.99936\end{array}$ \\
\hline$y=5$ & $\begin{array}{l}(1) \\
(2) \\
(3) \\
(4) \\
(5)\end{array}$ & $\begin{array}{l}0.90297 \\
0.90268 \\
0.99969 \\
0.90283 \\
0.99985\end{array}$ & $\begin{array}{l}0.14657 \\
0.14653 \\
0.99975 \\
0.14655 \\
0.99986\end{array}$ & $\begin{array}{l}0.00386 \\
0.00386 \\
0.99986 \\
0.00386 \\
0.99989\end{array}$ & $\begin{array}{l}0.00010 \\
0.00010 \\
0.99995 \\
0.00010 \\
0.99990\end{array}$ \\
\hline$y=\infty$ & $\begin{array}{l}(1) \\
(2) \\
(3) \\
(4) \\
(5)\end{array}$ & $\begin{array}{l}0.90909 \\
0.90909 \\
1.00000 \\
0.90909 \\
1.00000\end{array}$ & $\begin{array}{l}0.14756 \\
0.14757 \\
1.00006 \\
0.14757 \\
1.00001\end{array}$ & $\begin{array}{l}0.00389 \\
0.00389 \\
1.00017 \\
0.00389 \\
1.00004\end{array}$ & $\begin{array}{l}0.00010 \\
0.00010 \\
1.00045 \\
0.00010 \\
1.00012\end{array}$ \\
\hline
\end{tabular}

TABLE 2

INDIVIDUAL CLAIM AMOUNTS ARE EXPONENTIALLY DISTRIBUTED AND THE PREMIUM LOADING FACTOR IS 0.2

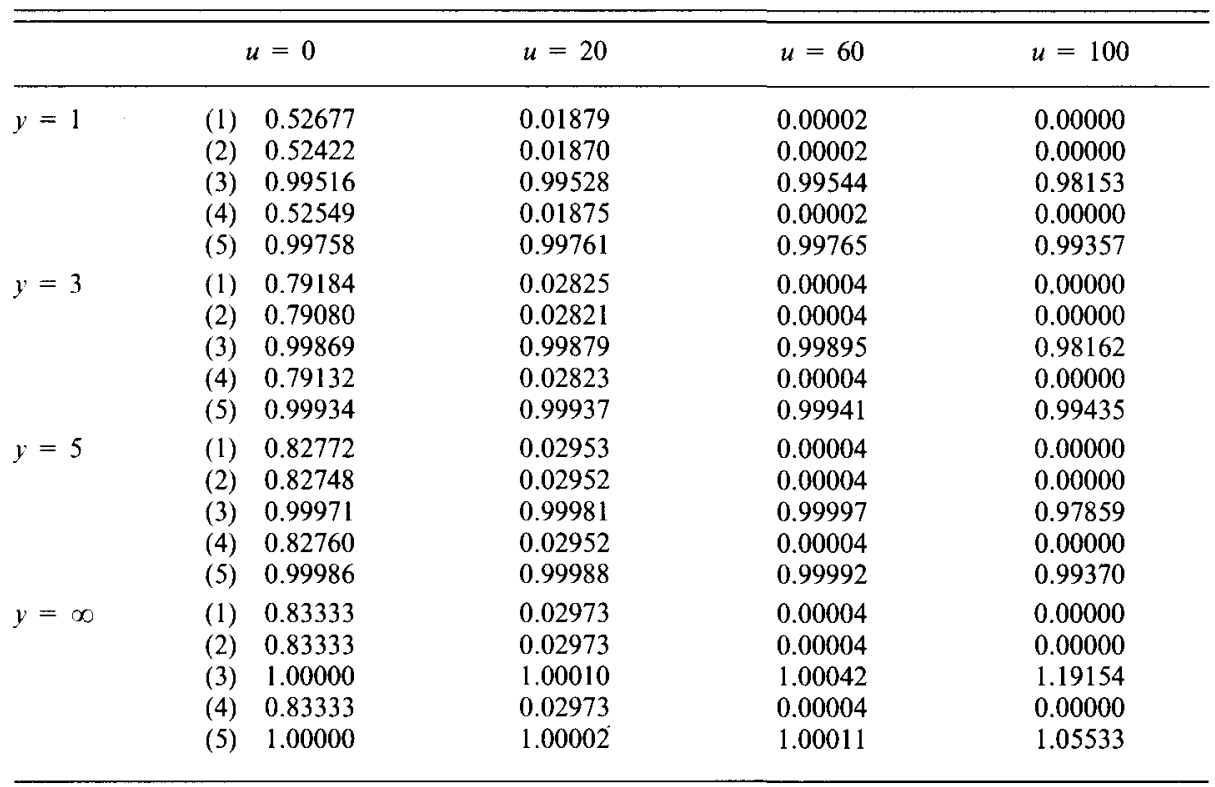


In all of our numerical examples, the discretization of the individual claim amount distribution is the same as that given by DE VYLDER and GoovaERTS (1988, Section 8).

We note the following points about Tables 1 and 2 :

(i) For all combinations of $u$ and $y$, we get a better approximation to ${ }_{0} G(u, y)$ when $\beta=100$. We commented in Section 2 that this is what we expected. The approximate values are less than the exact values for finite values of $y$, but in most cases are very close to the exact ones. For a given value of $u$, the approximations generally improve (i.e. the ratio becomes closer to one) as $y$ increases.

(ii) For a given value of $y$, the ratios do not always increase as $u$ increases. An example is when $y=3$ in Table 2. This, however, seems unimportant, particularly in Table 2 where the values of ${ }_{0} G(u, y)$ are so small for the larger values of $u$. The approximate values in Table 2 agree to five decimal places with the exact ones when $u \geq 40$ and $\beta=100$.

(iii) For each value of $u,{ }_{0} G(u, 5)$ is fairly close to the ultimate ruin probability.

Tables 3 and 4 show values for ${ }_{0} G(0, y)$ when individual claims amounts follow a Pareto distribution with distribution function

$$
B(x)=1-(1+x)^{-2}
$$

For this distribution, we can calculate exact values for ${ }_{0} G(u, y)$ only when $u=0$, and by integrating equation (3.6) we find that

$$
{ }_{0} G(0, y)=\frac{1}{1+\theta} \frac{y}{1+y}
$$

In Table 3 the premium loading factor is $10 \%$ and in Table 4 it is $20 \%$. The key to these tables is the same as for Tables 1 and 2, and the same method as above has been used to discretize the individual claim amount distribution.

TABLE 3

Individual Claim AMOUNTS have a PARETo distribution, THE PREMIUM LOADING FACTOR IS 0.1 AND THE INITIAL SURPLUS IS 0

\begin{tabular}{lllll}
\hline & $y=1$ & $y=5$ & $y=10$ & $y=\infty$ \\
\hline$(1)$ & 0.45455 & 0.75758 & 0.82645 & 0.90909 \\
$(2)$ & 0.45278 & 0.75712 & 0.82630 & 0.90909 \\
$(3)$ & 0.99612 & 0.99940 & 0.99982 & 1.00000 \\
$(4)$ & 0.45366 & 0.75735 & 0.82637 & 0.90909 \\
$(5)$ & 0.99806 & 0.99970 & 0.99991 & 1.00000 \\
\hline
\end{tabular}


THE PROBABILITY AND SEVERITY OF RUIN IN FINITE AND INFINITE TIME 185

TABLE 4

Individual Claim amounts have a Pareto distribution, THE PREMIUM LOADING FACTOR IS 0.2 AND THE INITIAL SURPLUS IS 0

\begin{tabular}{lllll}
\hline \hline & $y=1$ & $y=5$ & $y=10$ & $y=\infty$ \\
\hline$(1)$ & 0.41667 & 0.69444 & 0.75758 & 0.83333 \\
$(2)$ & 0.41518 & 0.69406 & 0.75745 & 0.83333 \\
$(3)$ & 0.99644 & 0.99945 & 0.99983 & 1.00000 \\
$(4)$ & 0.41593 & 0.69425 & 0.75751 & 0.83333 \\
$(5)$ & 0.99822 & 0.99973 & 0.99992 & 1.00000 \\
\hline
\end{tabular}

We note the following points about Tables 3 and 4 :

(i) The approximations are better when $\beta=100$. Also, for a given value of $\beta$, the ratios become closer to one as $y$ increases.

(ii) As in Tables 1 and 2, the approximate values are less than the exact values for finite values of $y$ and are closer to the exact values when $\beta=100$. The difference between exact and approximate values is small in most cases.

Tables 5 and 6 show approximate values of ${ }_{0} G(u, y)$ when individual claims have the Pareto distribution given by (4.1) and when $u>0$. In this situation, we cannot calculate exact values of ${ }_{0} G(u, y)$. In Table 5 the premium loading factor is 0.1 and in Table 6 it is 0.2 . The key to Tables (5) and (6) is as follows :

(1) denotes the approximation to ${ }_{0} G(u, y)$, calculated using (3.5) and (3.1), with the parameter $\beta=50$,

(2) as (1), but with $\beta=100$.

We note that the pattern of results in Tables 5 and 6 is the same as that in Tables 1 and 2. In particular, for finite values of $y$, the approximations are

TABLE 5

Individual Claim amounts have a Pareto distribution AND THE PREMIUM LOADING FACTOR IS 0.1

\begin{tabular}{|c|c|c|c|}
\hline & $u=20$ & $u=100$ & $u=200$ \\
\hline$y=1$ & $\begin{array}{ll}\text { (1) } & 0.07966 \\
\text { (2) } & 0.07982\end{array}$ & $\begin{array}{l}0.01289 \\
0.01292\end{array}$ & $\begin{array}{l}0.00359 \\
0.00359\end{array}$ \\
\hline$y=5$ & $\begin{array}{ll}\text { (1) } & 0.21114 \\
\text { (2) } & 0.21124\end{array}$ & $\begin{array}{l}0.03591 \\
0.03593\end{array}$ & $\begin{array}{l}0.01013 \\
0.01014\end{array}$ \\
\hline$y=10$ & $\begin{array}{ll}\text { (1) } & 0.28207 \\
\text { (2) } & 0.28213\end{array}$ & $\begin{array}{l}0.05068 \\
0.05069\end{array}$ & $\begin{array}{l}0.01455 \\
0.01455\end{array}$ \\
\hline$y=\infty$ & $\begin{array}{ll}\text { (1) } & 0.49815 \\
\text { (2) } & 0.49814\end{array}$ & $\begin{array}{l}0.16486 \\
0.16486\end{array}$ & $\begin{array}{l}0.07633 \\
0.07632\end{array}$ \\
\hline
\end{tabular}


TABLE 6

Individual claim amounts have a Pareto distribution AND THE PREMIUM LOADING FACTOR IS 0.2

\begin{tabular}{|c|c|c|c|}
\hline & $u=20$ & $u=100$ & $u=200$ \\
\hline$y=1$ & $\begin{array}{ll}\text { (1) } & 0.04170 \\
\text { (2) } & 0.04178\end{array}$ & $\begin{array}{l}0.00343 \\
0.00344\end{array}$ & $\begin{array}{l}0.00079 \\
0.00080\end{array}$ \\
\hline$y=5$ & $\begin{array}{ll}\text { (1) } & 0.11546 \\
\text { (2) } & 0.11551\end{array}$ & $\begin{array}{l}0.01027 \\
0.01027\end{array}$ & $\begin{array}{l}0.00242 \\
0.00242\end{array}$ \\
\hline$y=10$ & $\begin{array}{ll}\text { (1) } & 0.15818 \\
\text { (2) } & 0.15821\end{array}$ & $\begin{array}{l}0.01528 \\
0.01528\end{array}$ & $\begin{array}{l}0.00370 \\
0.00370\end{array}$ \\
\hline$y=\infty$ & $\begin{array}{ll}\text { (1) } & 0.30054 \\
\text { (2) } & 0.30054\end{array}$ & $\begin{array}{l}0.06915 \\
0.06915\end{array}$ & $\begin{array}{l}0.03114 \\
0.03114\end{array}$ \\
\hline
\end{tabular}

larger when $\beta=100$, but are very close to each other for most combinations of $u$ and $y$. One interesting point about Tables 5 and 6 is that values of ${ }_{0} G(u, 10)$ are not at all close to the values of ${ }_{0} G(u, \infty)$.

Unfortunately, for larger values of $u$ than those tabulated, this algorithm appears to be unstable, giving probabilities outside the interval $[0,1]$ for both distributions. Dickson and WATERS (1991, Sections 5 and 7) present a pragmatic approach to the problem of instability which can also be adopted for this algorithm.

In terms of numerical accuracy, there is little to choose between this algorithm to calculate ${ }_{0} G(u, y)$ and the one presented by DickSON (1989). A major difference between the algorithms is that the algorithm in Section 3 is for a fixed value of $y$ whereas Dickson's (1989) algorithm is for a fixed value of $u$. A second difference is that to apply DiCKSON's (1989) algorithm, calculated values for ultimate survival probabilities in our initial model are required to calculate ${ }_{0} G(u, y)$. The above algorithm works in the opposite way in the sense that the ultimate ruin probability is just the limiting value of ${ }_{0} G(u, y)$. A similarity between the algorithms is that to perform the recursive calculations in each case values from a compound distribution have to be calculated: for the algorithm in Section 3, values from a compound Poisson distribution are required; in Dickson's (1989) paper, values of the ultimate survival probability were required and were approximated using values from a compound geometric distribution (see PANJER (1986)). Despite these similarities, we have found that the algorithm in Section 3 generally requires less computer time.

\section{THE PROBABILITY AND SEVERITY OF RUIN IN FINITE TIME}

In this section we present a recursive algorithm for the calculation of $G(u, y, t)$, where $u$ is a non-negative integer and $y$ and $t$ are positive integers. Our 
algorithm is based on an algorithm presented by DE VYLDER and GoovaerTs (1988) for the calculation of finite time ruin probabilities. (See Dickson and WATERS (1991, Section 2) for comments on this algorithm.)

Our algorithm, which is derived by considering the aggregate claims at the end of the first time unit, is as follows:

$$
\begin{aligned}
& G(u, y, 1)=F(u+y)-F(u) \\
& G(u, y, t)=\sum_{j=0}^{u} f_{j} G(u+1-j, y, t-1)+G(u, y, 1) \\
& \text { for } \begin{aligned}
u & =0,1,2, \ldots \\
y & =1,2,3, \ldots \\
t & =2,3, \ldots
\end{aligned}
\end{aligned}
$$

We can use (5.1) to calculate $G(u, y, t)$ by calculating successively:

$$
\begin{array}{lll}
G(w, y, 1) & \text { for } & w=1,2, \ldots, u+t-1 \\
G(w, y, 2) & \text { for } & w=1,2, \ldots, u+t-2 \\
\ldots & & \\
G(w, y, t-1) & \text { for } & w=1,2, \ldots, u+1
\end{array}
$$

and finally $G(u, y, t)$.

We can reduce the number of calculations involved in this procedure by discarding very small values of $f_{k}$. This was a device used by DE VYLDER and GoovaerTs (1988, Section 5). Let $\varepsilon$ be some suitably small positive number. We define a sequence $\left\{f_{j}^{c}\right\}_{j=0}^{\infty}$ as follows:

$$
\begin{array}{lll}
f_{j}^{\varepsilon}=f_{j} & \text { if } & F(j) \leq 1-\varepsilon \\
f_{j}^{\varepsilon}=0 & \text { if } & F(j)>1-\varepsilon
\end{array}
$$

It follows immediately from (5.2) that for any $j \geq 0$ :

$$
\sum_{k=0}^{j} f_{k}-\varepsilon \leq \sum_{k=0}^{j} f_{k}^{\varepsilon} \leq \sum_{k=0}^{j} f_{k}
$$

We now define $G^{\varepsilon}(u, y, t)$ as follows:

$$
\begin{aligned}
& G^{\varepsilon}(u, y, 1)=F(u+y)-F(u) \quad \text { if } \quad F(u) \leq 1-\varepsilon \\
& =0 \quad \text { if } F(u)>1-\varepsilon
\end{aligned}
$$

and for $t=2,3,4, \ldots$

$$
G^{\varepsilon}(u, y, t)=\sum_{j=0}^{u} f_{j}^{\varepsilon} G^{\varepsilon}(u+1-j, y, t-1)+G^{\varepsilon}(u, y, 1)
$$

The advantage of using (5.4) and (5.5) rather than (5.1) is that the summation in (5.5) is restricted to those values of $j$ for which $f_{j}^{\varepsilon}$ is not zero. 
The error introduced by using (5.4) and (5.5) can be bounded as shown by the following Result (see De VyLDer and GoovaerTs (1988, Section 5)):

\section{Result :}

$$
G(u, y, 1)-\varepsilon \leq G^{\varepsilon}(u, y, 1) \leq G(u, y, 1)
$$

and for $t=2,3, \ldots$

$$
G(u, y, t)-2 t \varepsilon \leq G^{\varepsilon}(u, y, t) \leq G(u, y, t)
$$

Proof: The second inequality in (5.6) follows from the definition of $G^{\varepsilon}(u, y, 1)$; the second inequality in (5.7) follows by induction from (5.6) and from noting that $f_{j}^{\varepsilon} \leq f_{j}$ for all $j$.

To prove the first inequality in (5.6) note that $G(u, y, 1)$ equals $G^{\varepsilon}(u, y, 1)$ unless $F(u)$ is greater than $(1-\varepsilon)$, in which case the former is less than $\varepsilon$ and the latter is zero.

It remains to prove that:

$$
G(u, y, t)-2 t \varepsilon \leq G^{\varepsilon}(u, y, t)
$$

First note that (5.8) is true for $t=1$ (and for all $u$ and $y$ ). Suppose it is true for a particular value of $t$ (and for all $u$ and $y$ ). Then:

$$
\begin{aligned}
G(u, y, t+1)-G^{\varepsilon}(u, y, t+1) & =\sum_{j=0}^{u}\left\{f_{j} G(u+1-j, y, t)-f_{j}^{\varepsilon} G^{\varepsilon}(u+1-j, y, t)\right\} \\
& +G(u, y, 1)-G^{\varepsilon}(u, y, 1) \\
& \leq \sum_{j=0}^{u}\left\{f_{j}-f_{j}^{\varepsilon}\right\} G(u+1-j, y, t) \\
& +\sum_{j=0}^{u} f_{j}^{\varepsilon}\left\{G(u+1-j, y, t)-G^{\varepsilon}(u+1-j, y, t)\right\}+\varepsilon \\
& \leq \sum_{j=0}^{u}\left\{f_{j}-f_{j}^{\varepsilon}\right\}+2 \varepsilon t \sum_{j=0}^{u} f_{j}^{\varepsilon}+\varepsilon \\
& \leq \varepsilon+2 \varepsilon t+\varepsilon=2 \varepsilon(t+1)
\end{aligned}
$$

and (5.8) follows by induction.

\section{NUMERICAL EXAMPLES}

Table 7 shows approximate values of ${ }_{0} G(u, y, t)$ and, for comparison, of ${ }_{0} G(u, y)$ and ${ }_{0} \psi(u, t)$ for various combinations of $u, y$ and $t$ and two different 
TABLE 7

Approximate values of ${ }_{0} \mathrm{G}(u, y, t)$ and, for comparison, of ${ }_{0} G(u, y)$ and ${ }_{0} \psi(u, t)$. THE PREMIUM LOADING FACTOR IS 0.1

\begin{tabular}{|c|c|c|c|c|c|c|c|c|}
\hline \multirow[b]{2}{*}{$t$} & \multirow[b]{2}{*}{$u$} & \multirow[b]{2}{*}{$y$} & \multicolumn{3}{|c|}{ Exponential claim amounts } & \multicolumn{3}{|c|}{ Pareto claim amounts } \\
\hline & & & $\begin{array}{c}(1) \\
{ }_{0} G(u, y, t)\end{array}$ & $\begin{array}{c}(2) \\
{ }_{0} G(u, y)\end{array}$ & $\begin{array}{c}(3) \\
{ }_{0} \psi(u, t)\end{array}$ & $\begin{array}{c}(4) \\
{ }_{0} G(u, y, t)\end{array}$ & $\begin{array}{c}(5) \\
{ }_{0} G(u, y)\end{array}$ & $\begin{array}{c}(6) \\
{ }_{0} \psi(u, t)\end{array}$ \\
\hline \multirow[t]{3}{*}{10} & 0 & 1 & 0.4899 & 0.5747 & 0.7854 & 0.3988 & 0.4501 & 0.6939 \\
\hline & & 3 & 0.7436 & 0.8638 & 0.7854 & 0.5800 & 0.6797 & 0.6939 \\
\hline & & 5 & 0.7795 & 0.9030 & 0.7854 & 0.6318 & 0.7564 & 0.6939 \\
\hline \multirow[t]{3}{*}{10} & 10 & 1 & 0.0198 & 0.2315 & 0.0319 & 0.0225 & 0.1247 & 0.0932 \\
\hline & & 3 & 0.0301 & 0.3480 & 0.0319 & 0.0452 & 0.2500 & 0.0932 \\
\hline & & 5 & 0.0315 & 0.3638 & 0.0319 & 0.0575 & 0.3198 & 0.0932 \\
\hline \multirow[t]{3}{*}{10} & 20 & 1 & 0.0002 & 0.0933 & 0.0004 & 0.0044 & 0.0792 & 0.0278 \\
\hline & & 3 & 0.0004 & 0.1402 & 0.0004 & 0.0094 & 0.1619 & 0.0278 \\
\hline & & 5 & 0.0004 & 0.1466 & 0.0004 & 0.0126 & 0.2108 & 0.0278 \\
\hline \multirow[t]{3}{*}{100} & 0 & 1 & 0.5552 & 0.5747 & 0.8900 & 0.4404 & 0.4501 & 0.8432 \\
\hline & & 3 & 0.8426 & 0.8638 & 0.8900 & 0.6597 & 0.6797 & 0.8432 \\
\hline & & 5 & 0.8833 & 0.9030 & 0.8900 & 0.7305 & 0.7564 & 0.8432 \\
\hline \multirow[t]{3}{*}{100} & 10 & 1 & 0.1625 & 0.2315 & 0.2606 & 0.0898 & 0.1247 & 0.3820 \\
\hline & & 3 & 0.2466 & 0.3480 & 0.2606 & 0.1784 & 0.2500 & 0.3820 \\
\hline & & 5 & 0.2585 & 0.3638 & 0.2606 & 0.2264 & 0.3198 & 0.3820 \\
\hline \multirow[t]{3}{*}{100} & 20 & 1 & 0.0376 & 0.0933 & 0.0604 & 0.0407 & 0.0792 & 0.2122 \\
\hline & & 3 & 0.0571 & 0.1402 & 0.0604 & 0.0829 & 0.1619 & 0.2122 \\
\hline & & 5 & 0.0599 & 0.1466 & 0.0604 & 0.1074 & 0.2108 & 0.2122 \\
\hline
\end{tabular}

claim amount distributions: the exponential distribution with mean 1 and the Pareto distribution specified by (4.1). Columns (1), (2) and (3) relate to the exponential claim amount distribution and columns (4), (5) and (6) give the corresponding information for the Pareto distribution. Throughout Table 7 the premium loading factor, $\theta$, has been taken to be 0.1 . The key to Table 7 is as follows :

(1) \& (4) denote the approximate values of ${ }_{0} G(u, y, t)$. The figures in these columns are values of $G^{\varepsilon}(u \beta, y \beta, t \beta(1+\theta))$ calculated using the algorithm in Section 5. The parameter $\beta$ has been taken to be 20 and, for a given value of $t$, the parameter $\varepsilon$ has been taken to be $10^{-3} /(2 t \beta(1+\theta))$ so that from $(5.6)$ and $(5.7)$ it can be seen that the difference between $G(u \beta, y \beta, t \beta(1+\theta))$ and $G^{\varepsilon}(u \beta, y \beta, t \beta(1+\theta))$ is at most $10^{-3}$.

(2) \& (5) denote the approximate values of ${ }_{0} G(u, y)$. The figures in these columns are values of $G(u \beta, y \beta)$ with $\beta$ taken to be 20 and these have been calculated as in Tables 1 and 5 .

(3) \& (6) denote the approximate values ${ }_{0} \psi(u, t)$. The figures in these columns are values of $\psi(u \beta, t \beta, t \beta(1+\theta))$ and have been calculated using the methods in Dickson and WATERS (1991, Sections 3, 4 and 8). 
There are two interesting comments to be made about the figures in Table 7.

The first is that for the exponential claim amount distribution the values of $G(u, 5, t)$ are reasonably close to the values of $\psi(u, t)$. This is not surprising since ruin must be caused by a single claim and the probability that a single claims exceeds 5 is very small $(0.006738)$. However, these values are not so close for the Pareto distribution and this is presumably because the probability that a single claim exceeds 5 is much larger in this case $(0.02778)$. The second point is that, apart from the case $u=0$, the values of $G(u, y, t)$ are not very close to the corresponding values of $G(u, y)$, even for the largest value of $t$ $(t=100)$ This means that, for these ranges of values of $u, y$ and $t$ and for these claim distributions, it would not be reasonable to use $G(u, y)$ as an approximation to $G(u, y, t)$.

\section{REFERENCES}

De Vylder, F. and Goovaerts, M. J. (1988) Recursive calculation of finite time ruin probabilities. Insurance: Mathematics and Economics 7, 1-8.

Dickson, D.C.M. (1989) Recursive calculation of the probability and severity of ruin. Insurance: Mathematics and Economics 8, 145-148.

Dickson, D.C.M. and WATERS, H.R. (1991) Recursive calculation of survival probabilities. ASTIN Bulletin 21, 199-221.

Dufresne, F. (1988) Distributions stationnaires d'un système bonus-malus et probabilité de ruine. ASTIN Bulletin 18, 31-46.

Gerber, H.U., Goovaerts, M.J. and KaAs, R. (1987) On the probability and severity of ruin. ASTIN Bulletin 17, 151-163.

Gerber, H. U. and Dufresne, F. (1988) The probability and severity of ruin for combinations of exponential claim amounts and their translations. Insurance: Mathematics and Economics 7 , 75-80.

PANJER, H.H. (1981) Recursive calculation of a family of compound distributions. ASTIN Bulletin 12, 22-26.

PANJER, H.H. (1986) Direct calculation of ruin probabilities. Journal of Risk and Insurance 53, 521-529.

David C. M. Dickson and Howard R. Waters

Department of Actuarial Mathematics and Statistics

Heriot-Watt University,

Edinburgh $\mathrm{EH} 14$ $4 \mathrm{AS}$

United Kingdom. 